\title{
The front-end Electronics for the LHCb upgrade.
}

\author{
Jan Buytaert (C.E.R.N.) \\ On behalf of the LHCb collaboration. \\ TWEPP 2010 Workshop, Aachen, \\ September 21, 2010
}


- The LHCb upgrade plan \& main issues.

- The new trigger/daq architecture.

- Overview of the subdetector modifications.

- Subdetector electronic developments.

- Summary. 
- The Plan:

\begin{tabular}{|c|c|c|c|c|}
\hline & E & $\begin{array}{c}\text { Peak } \& \\
\left(10^{32} \mathrm{~cm}^{-2} \mathrm{~s}^{-}\right. \\
1)\end{array}$ & 2 & \\
\hline 2011 & $7 \mathrm{TeV}$ & 2 & $1 \mathrm{fb}^{-1}$ & $=$ LHCb design luminosity ! \\
\hline \multicolumn{5}{|l|}{2012} \\
\hline 2013 & $>10 \mathrm{TeV}$ & 2 & $1 \mathrm{fb}^{-1}$ & \\
\hline 2014 & $>10 \mathrm{TeV}$ & 2 & $2 \mathrm{fb}^{-1}$ & \\
\hline 2015 & $>10 \mathrm{TeV}$ & 2 & $2 \mathrm{fb}^{-1}$ & Total $\int \mathcal{L} \sim 6 \mathrm{fb}^{-1}$ \\
\hline 2016 & & & & Install LHCb upgrade ! \\
\hline 2017 & $>10 \mathrm{TeV}$ & 10 & $10 \mathrm{fb}^{-1}$ & \\
\hline 2018 & $>10 \mathrm{TeV}$ & 10 & $10 \mathrm{fb}^{-1}$ & \\
\hline
\end{tabular}

- The increase in luminosity 'only' requires stronger beam squeezing, i.e. does not depend on any LHC upgrade.

- A 'letter of intent' will be submitted very soon to LHCC. 


\section{The occupancies.}

\begin{tabular}{|l|c|c|c|}
\hline Peak L $\left(10^{32} \mathrm{~cm}^{-2} \mathrm{~s}^{-1}\right)$ & 2 & 10 & 20 \\
\hline $\begin{array}{l}\text { Average \# of } \\
\text { interactions / crossing }\end{array}$ & 0.4 & 2.0 & 4.0 \\
\hline $\begin{array}{l}\text { Average \# of interactions/non- } \\
\text { empty crossing }\end{array}$ & 1.3 & 2.4 & 4.3 \\
\hline
\end{tabular}

- The occupancies will only increase by $\sim 2$ due to multiple interactions.

- The current detector granularities are

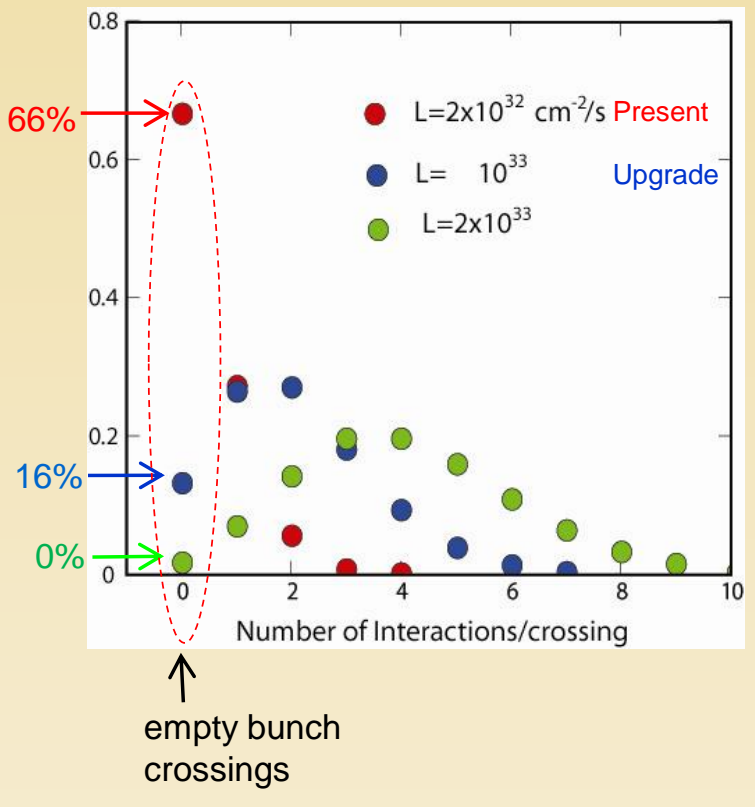
adequate and no major detector changes required.

- The exact beam conditions are under discussion with LHC. 


\section{L0 trigger efficiency.}

LHCb
IHCP

- Problem: the L0 hadron hardware trigger loses efficiency at higher luminosities. The event yield is even dropping...

- Because the DAQ event readout rate is limited to $1 \mathrm{MHz}$, the trigger thresholds must be raised ...

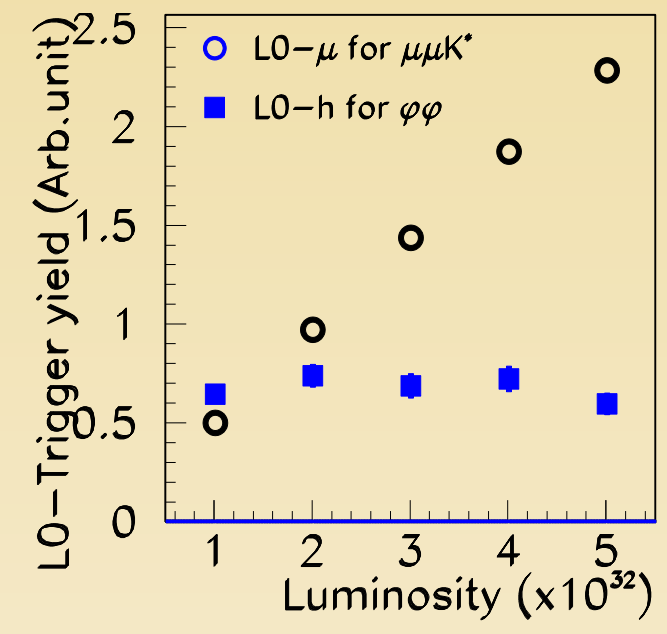

- 2 solutions:

- A more sophisticated hardware hadron trigger or

- No hardware trigger, increase the readout rate and implement trigger in a CPU farm !

$\square \quad$ Full detector information available.

- Flexible algorithm.

$\square \quad$ Double the hadron trigger efficiency .
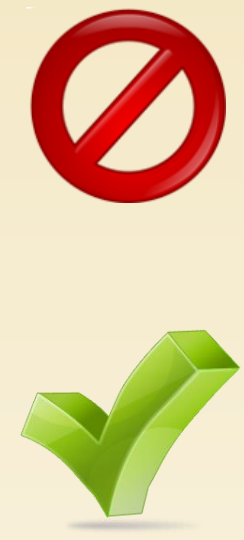


\section{New Trigger/DAQ architecture. IHCh}

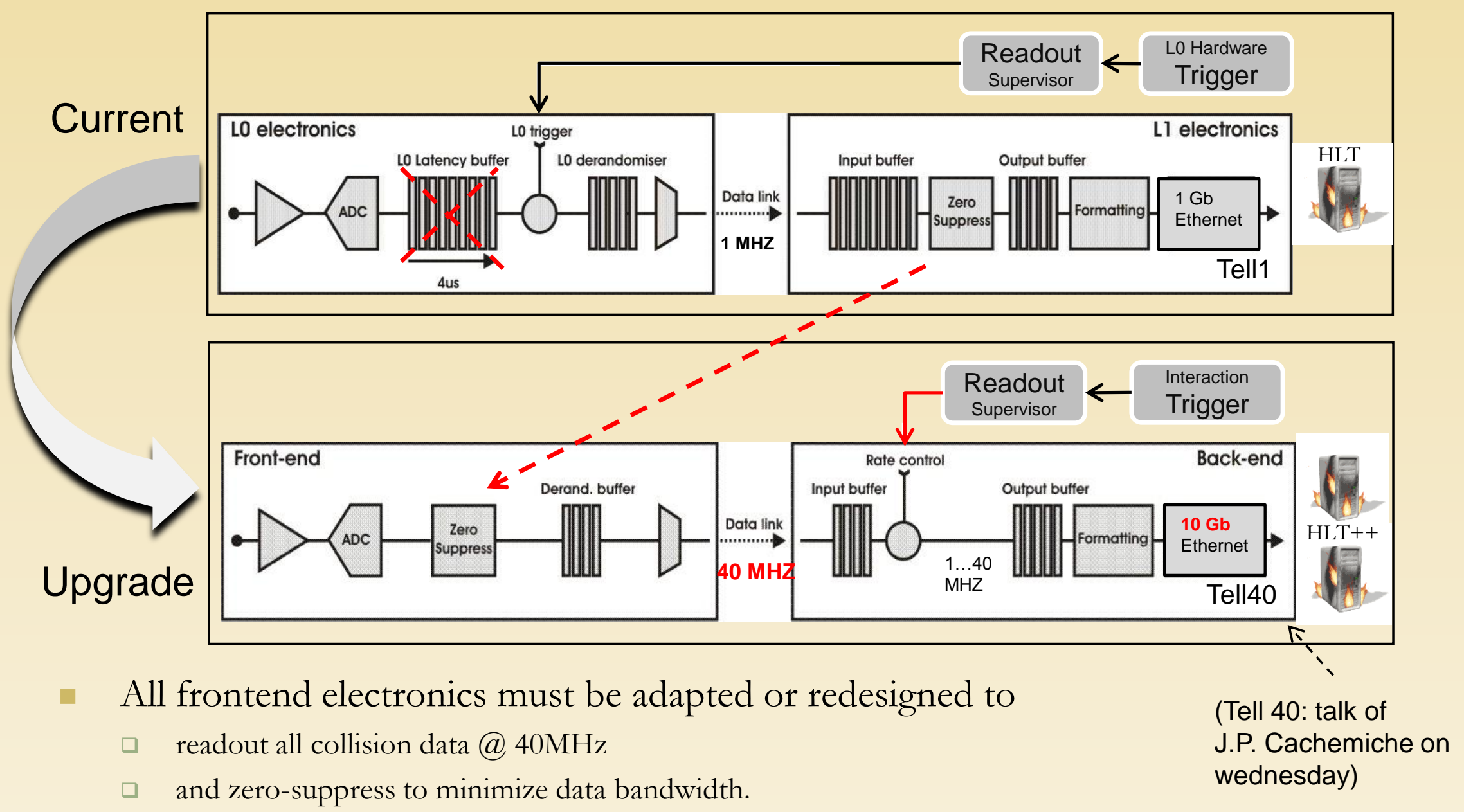

- The L0 hardware trigger is re-used to reduce the event rate to match the installed router and CPU farm capacity (staging). Initially run at $\sim 5 \mathrm{MHz}$. 


\section{Typical Subdetector readout \& control: LHCb}

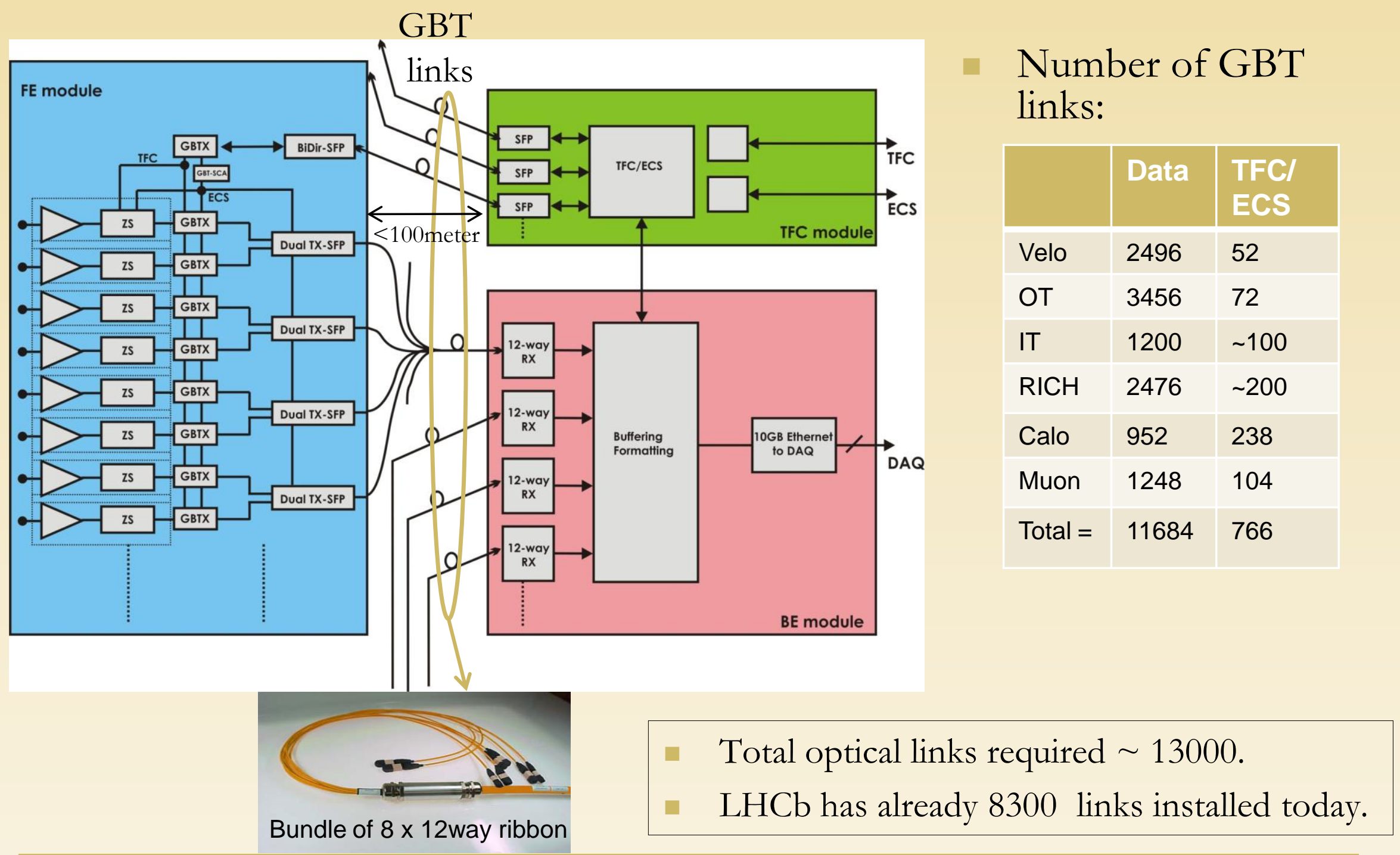




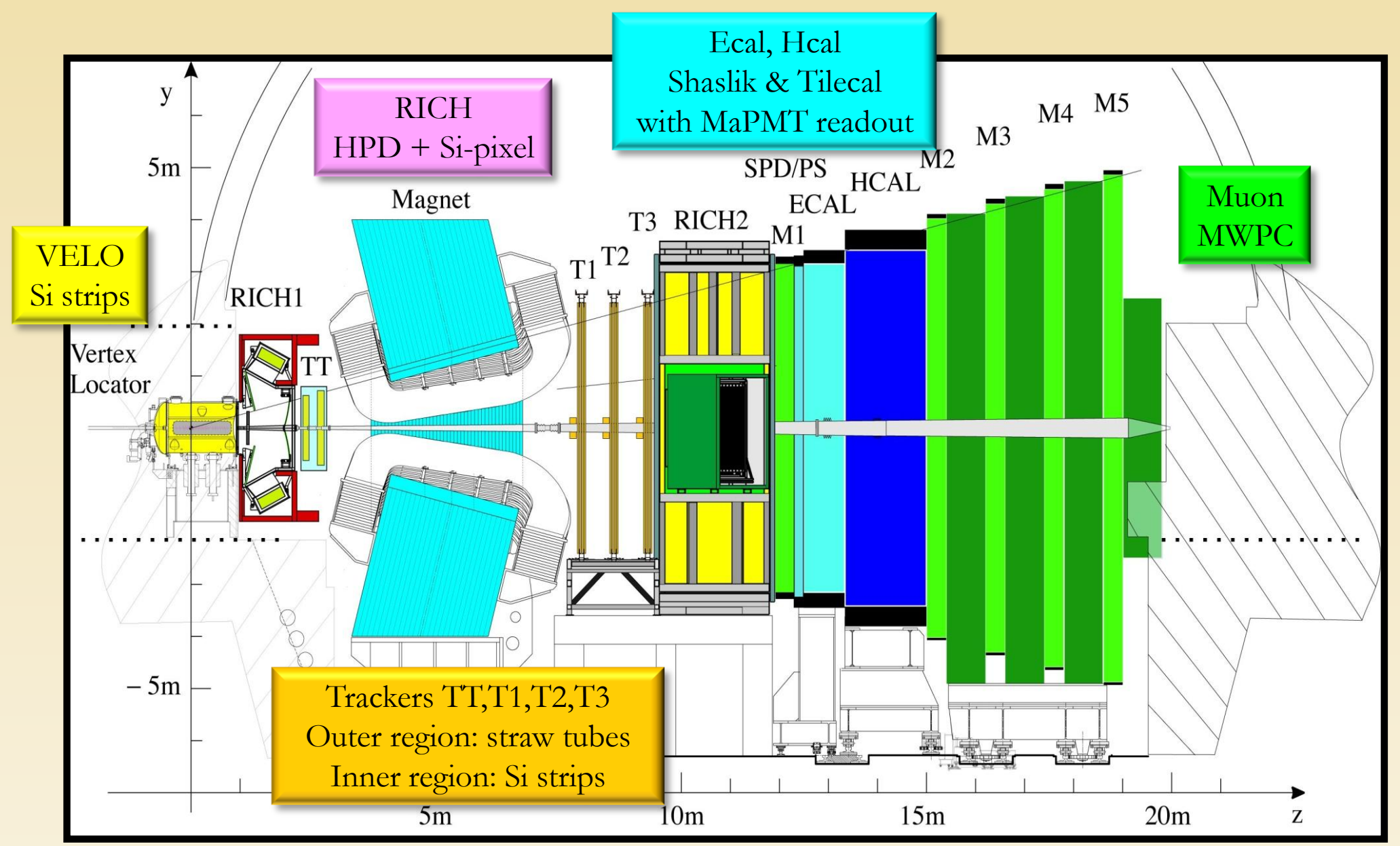

(See talk of K. Henessy on Friday). 


\section{Main detector modifications.}

\section{VELO and RICH:}

$\square$ Fullz new sensor modules and photon detectors (because they are highly integrated with the FE-electronics):

- VELO : pixel sensors will replace R-Phi strip sensors.

- RICH : multi-anode PMT's will replace Hybrid photon detector (HPD) tubes.

- Tracker :

- Inner Tracker : Keep current Si strip sensors or use scintillating fibers.

- Outer Tracker : Keep straw tubes and part of the FE electronics.

- Calorimeters:

- Keep MaPMT's, but at reduced HV to avoid ageing.

- $\quad$ - $>5 x$ lower signal gain $->$ to maintain $\mathrm{S} / \mathrm{N}$, need lower noise $->$ new amplifiers ...

$\square \quad$ Possibly remove pre-shower (PS) and scintillating pad (SPD).

- Muon:

- Detector and FE electronics stay unmodified.

- The M1 station will be removed. 


\section{VELO upgrade.}

Current VELO R\&Phi strips

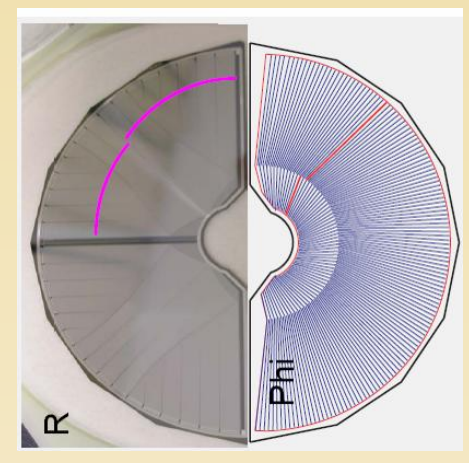

Current VELO module layout

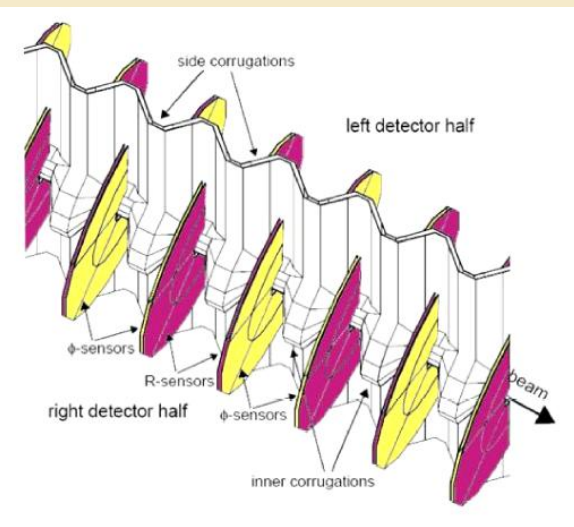

Upgrade : Pixel Sensor tile
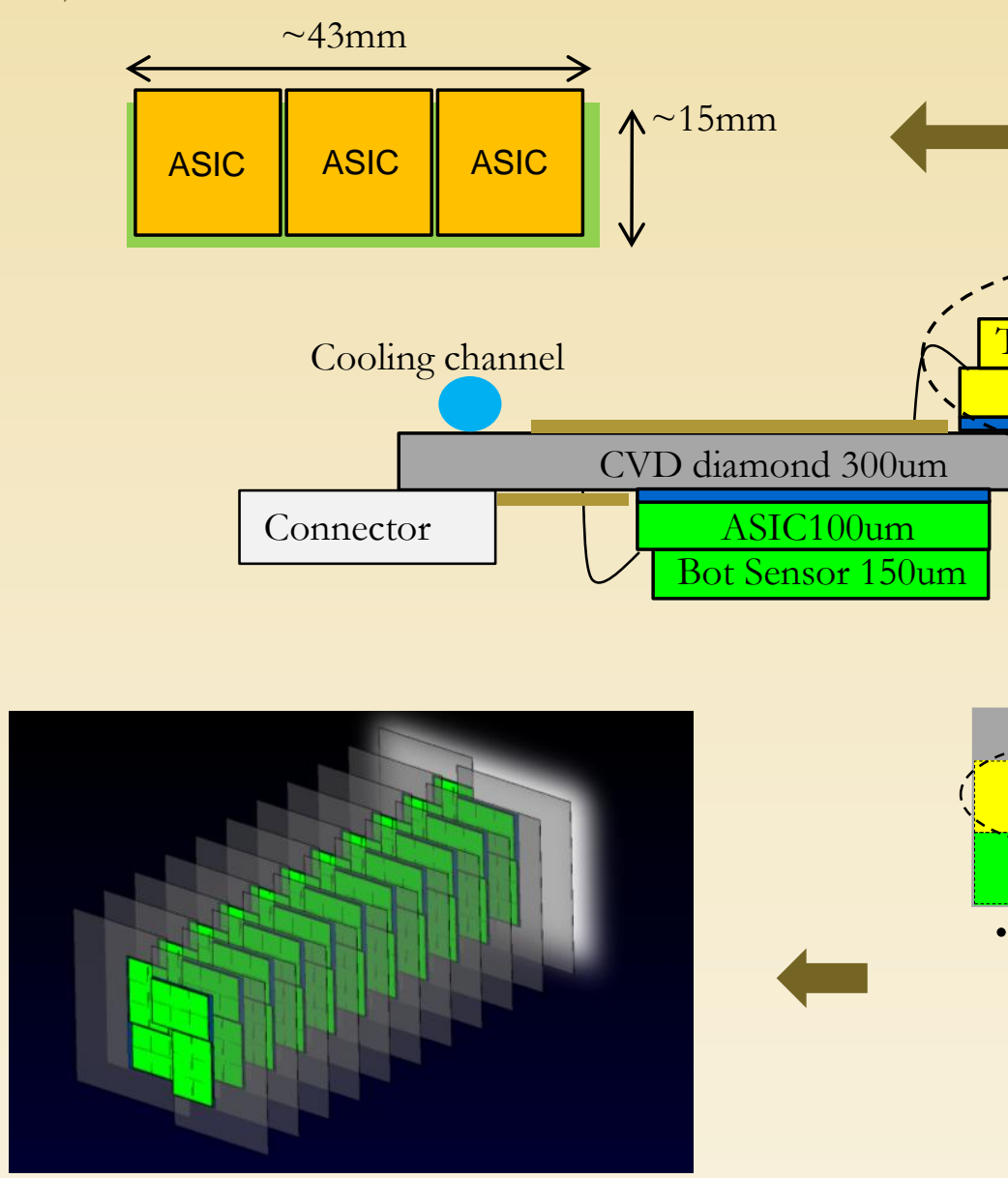

Pixel module

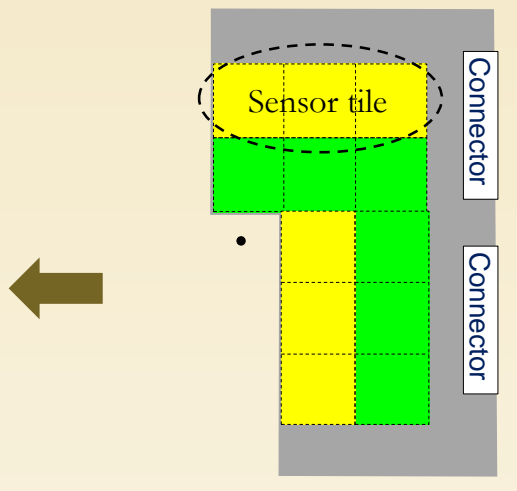


Design of a readout asic VELOPIX' in close collaboration with TimePix2.

- 256x256 pixel array, 55um x 55um pixel size.

- Minimal insensitive area $(\sim 5 \%)$

- Analog requirements identical.

- Simultaneous meaurement of Time-overthreshold and time identification of hits.

- Radiation hardness TID 400Mrad.

- Specific VELOPIX:

- Highest average particle rate is $200 \mathrm{MHz} / \mathrm{cm}^{2}$. $=>12 \mathrm{~Gb} / \mathrm{s}$ data generation rate /asic !

$\square$ Clustering and formatting in pixel.

- 'Superpixel' = group digital logic of $4 \mathrm{x} 4$ pixels in a single area.

- High speed column readout (8bit@40MHz).

- 4 multi-Gbit output links.

- Total power budget<3W@1.2 V

\begin{tabular}{l|c}
\hline Bipolar Input charge & \\
\hline Leakage current compensation & \\
\hline Peaking time & $\leq 25 \mathrm{~ns}$ \\
\hline Preamp output linear dynamic range & $<40 \mathrm{Ke}-$ \\
\hline ENC ( $\left.\sigma_{\text {ENC }}\right)$ & $\sim 75 \mathrm{e}-$ \\
\hline Detector capacitance & $<50 \mathrm{fF}$ \\
\hline Discriminator response time & $<2 \mathrm{~ns}$ \\
\hline Full chip minimum detectable charge & $<500 \mathrm{e}-$ \\
\hline Threshold spread after tuning & $<30 \mathrm{e}-$ \\
\hline Pixel analog power consumption @ 1.2V & $<15-20 \mu \mathrm{W}$ \\
\hline
\end{tabular}




\section{VELO upgrade.}

Module readout concept :

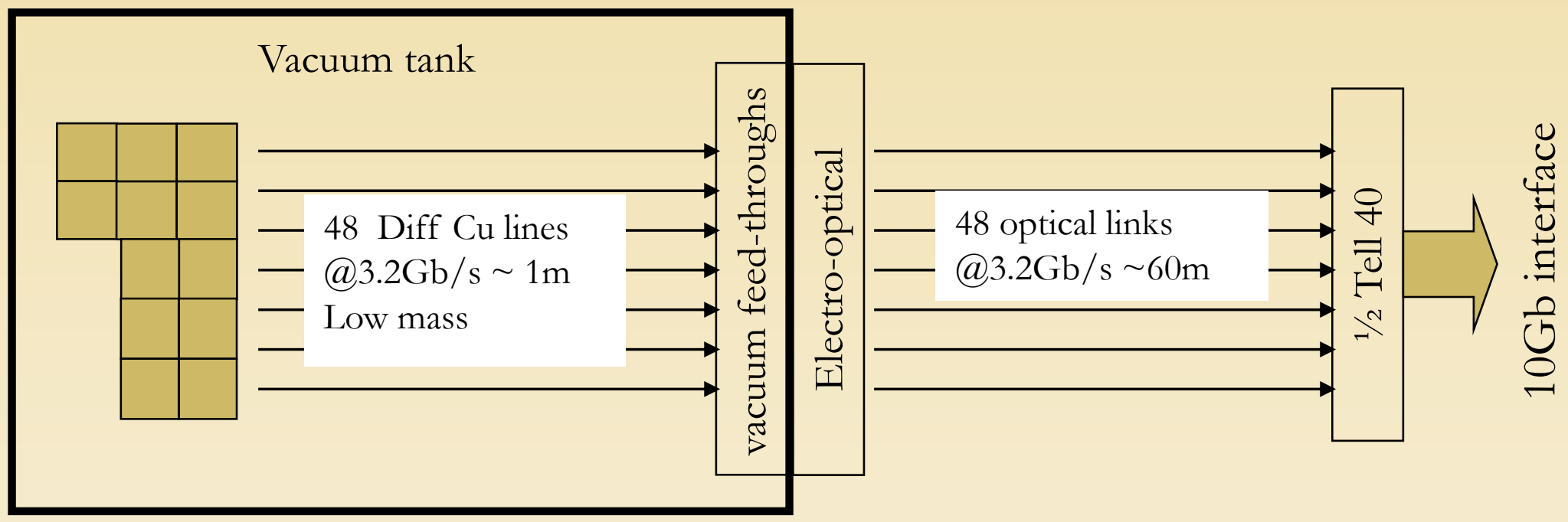

- Total of 52 modules in full system.

- Work is starting on the link technology. 


\section{RICH upgrade.}

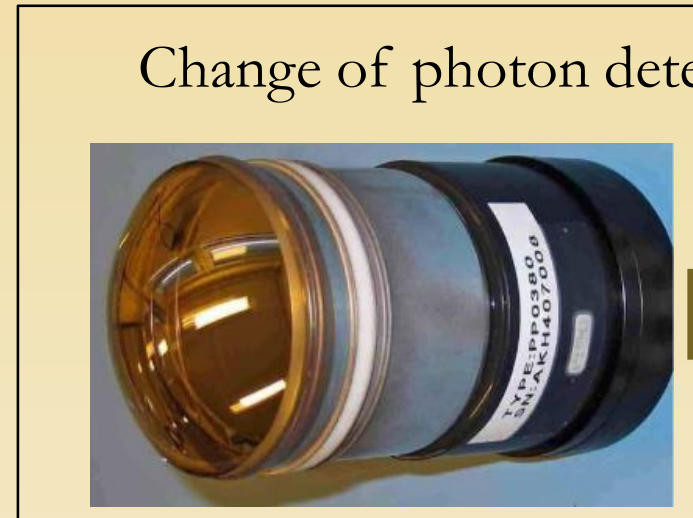

$32 \times 32$ pixels

Pixel size $2.5 \times 2.5 \mathrm{~mm} 2$

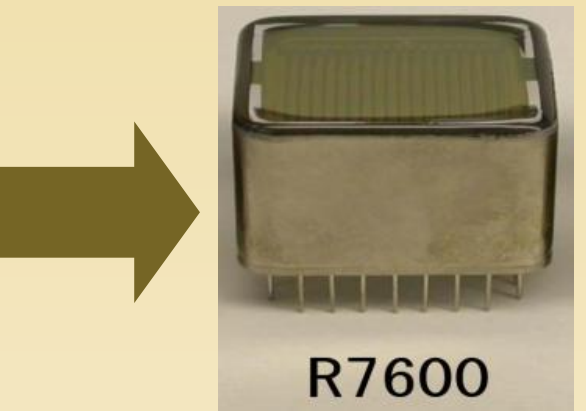

$8 \times 8$ pixels

Pixel size $2 \times 2 \mathrm{~mm} 2$
R7600 - Single Photon Signal at 950V

Larg pixel gain variation. $=>$ Electronic gain must be adjustable per pixel.

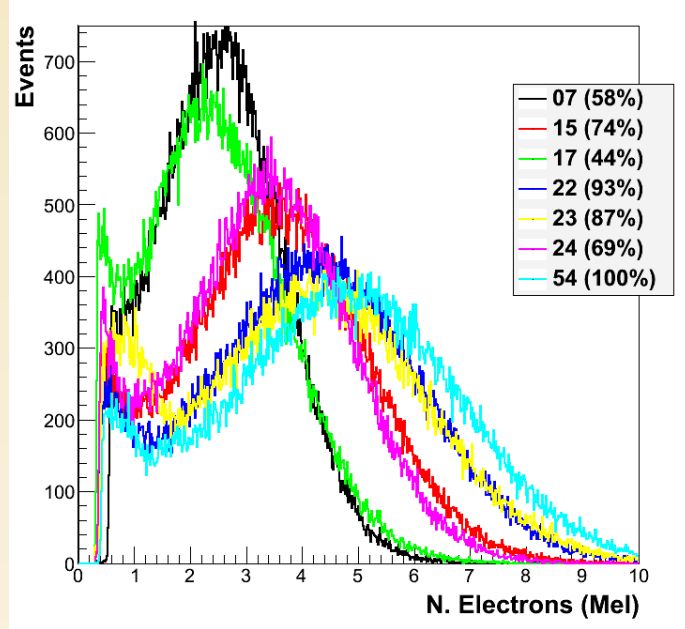

Inter-pixel crosstalk in $\mathrm{R} 7600$ is much less than in previous models :

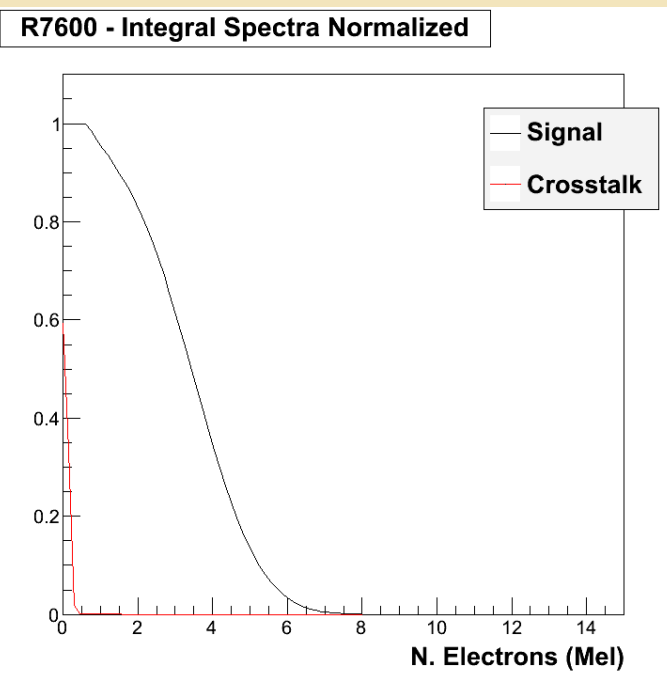




\section{RICH upgrade.}

\section{Discrete component prototyping:}

- 8-channel PCB

- Using SiGe npn transistors.

- Total power dissipation $<10 \mathrm{~mW} /$ channel

- Low noise $\sim 2.2 \mathrm{nV} / \sqrt{\mathrm{Hz}}$

- $\quad$ rise-time $=1.4 \mathrm{~ns}$, fall-time $=4 \mathrm{~ns}$

- Next:

- Start design of asic version.

- Prototype of digital functionality in FPGA.
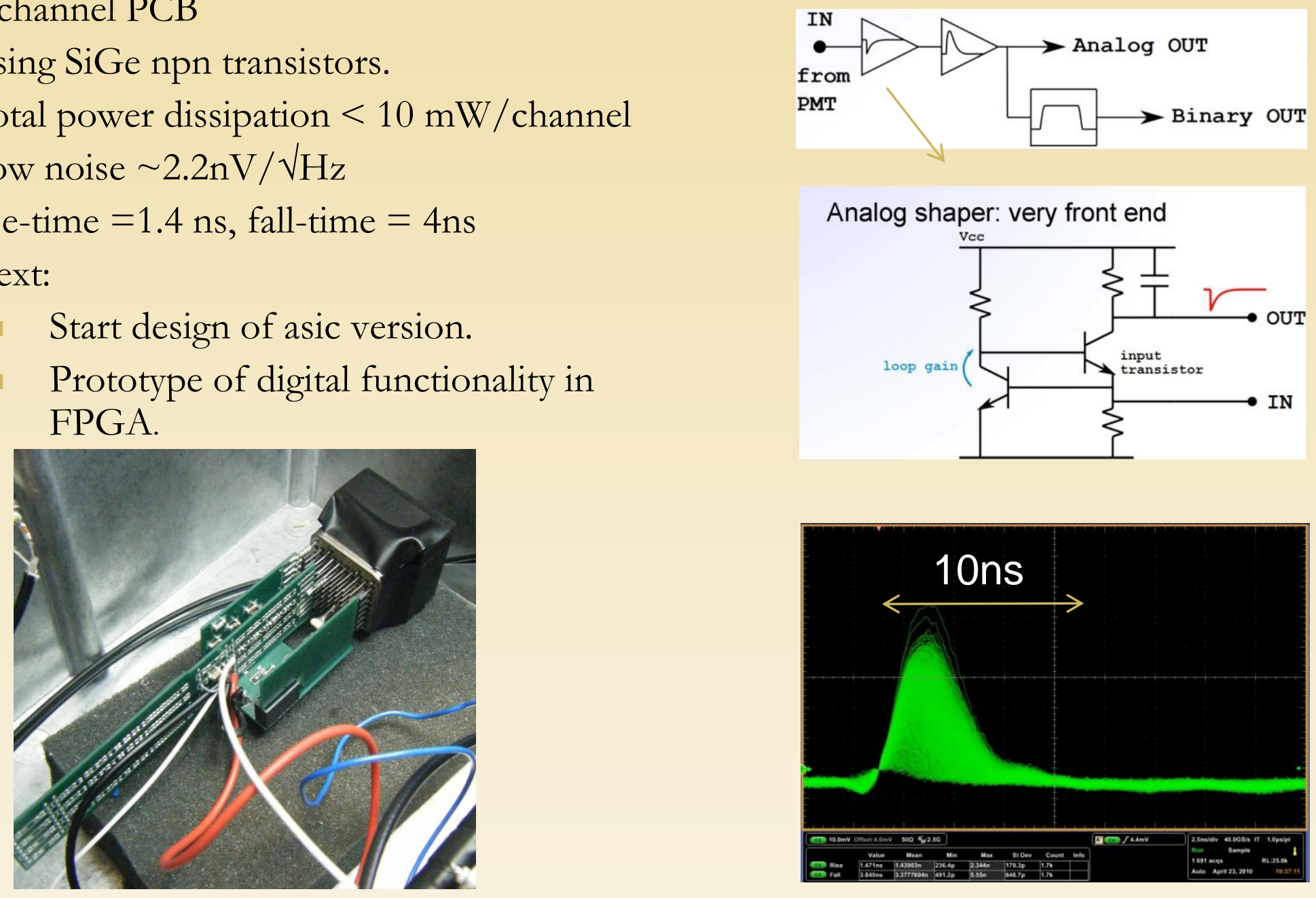

Analog shaper: very front end
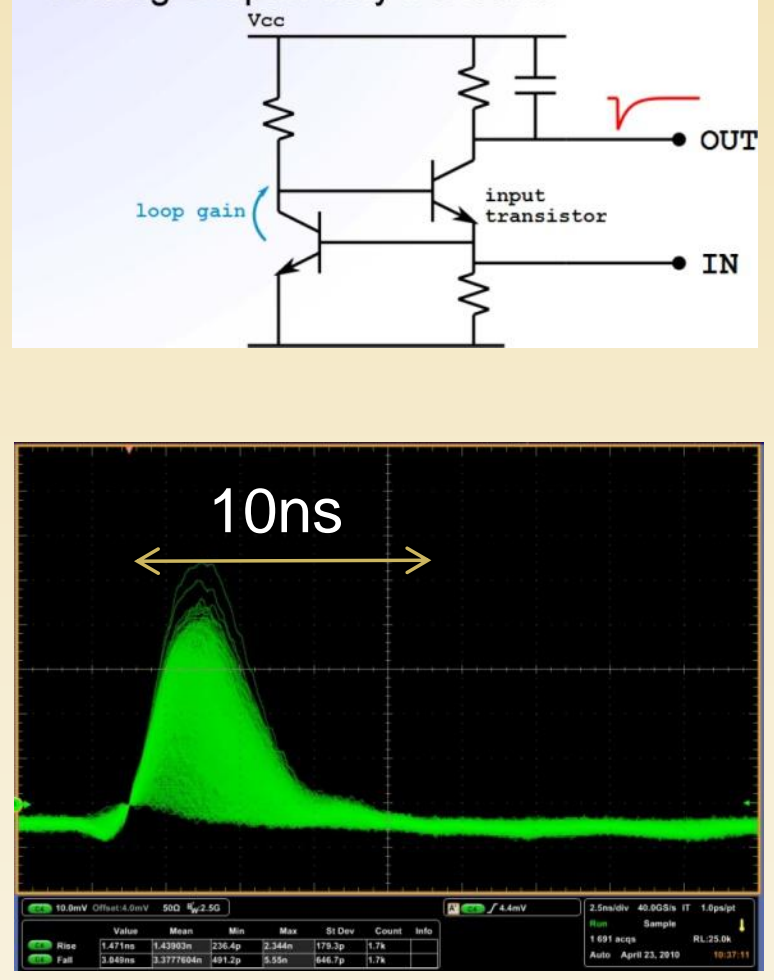
Current FE boards (432) $\quad \longrightarrow$ Upgrade
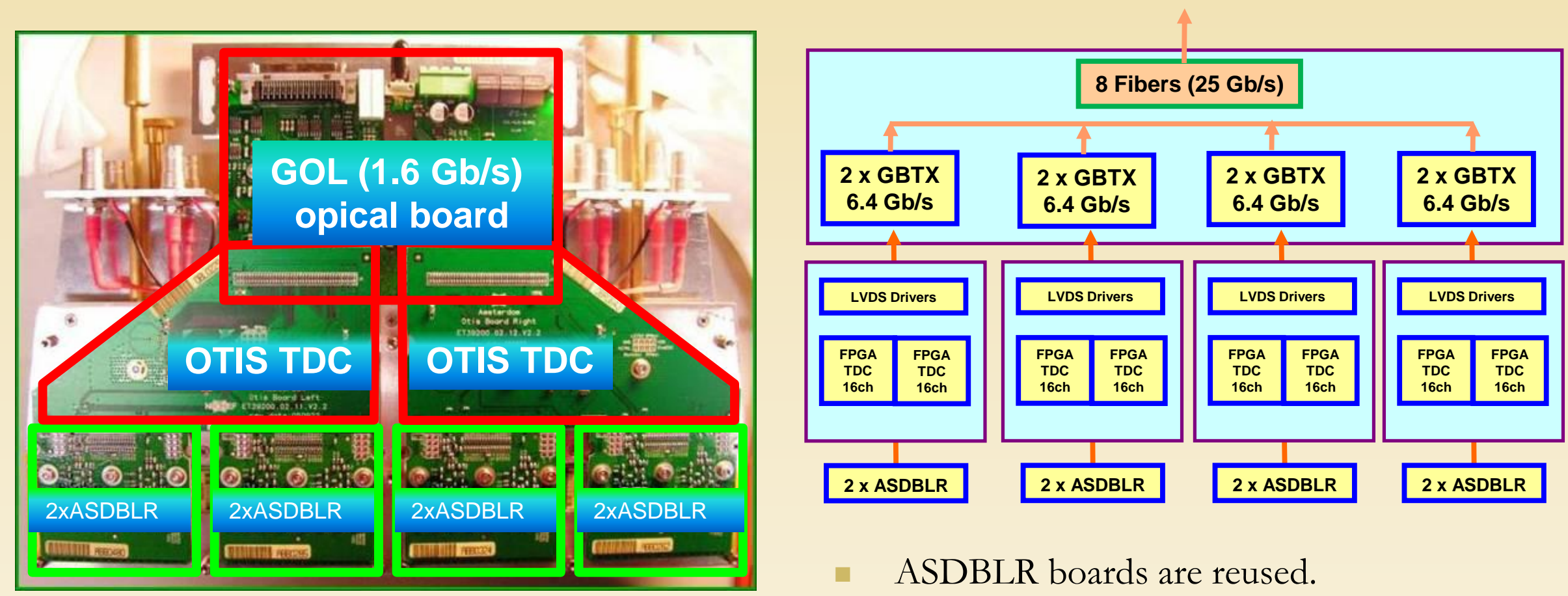

- ASDBLR boards are reused.

- OTIS TDC replaced by FPGA TDC.

1GOL replaced by 8 GBT 


\section{Outer Tracker upgrade.}

All logic is implemented in ACTEL Proasic3E FPGA for radiation tolerance .

- 16 Channel 4 bit TDC design finished.

- INL and DNL checked.

- Good temperature stability checked.

- Zero Suppression and data formatting.

- Output on parallel bus (20 bit@160 MHz) to GBT.

- I2C interface for configuration.

\section{Prototyping :}

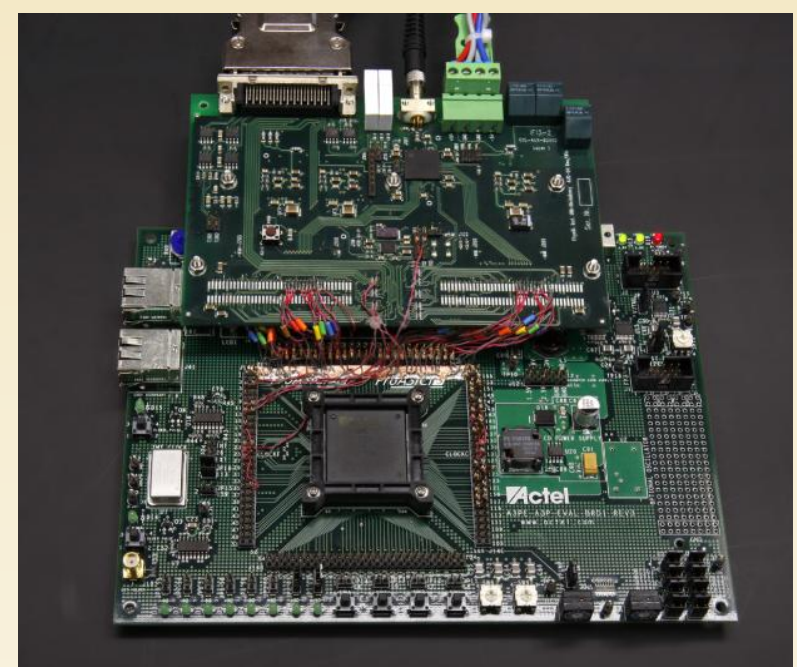

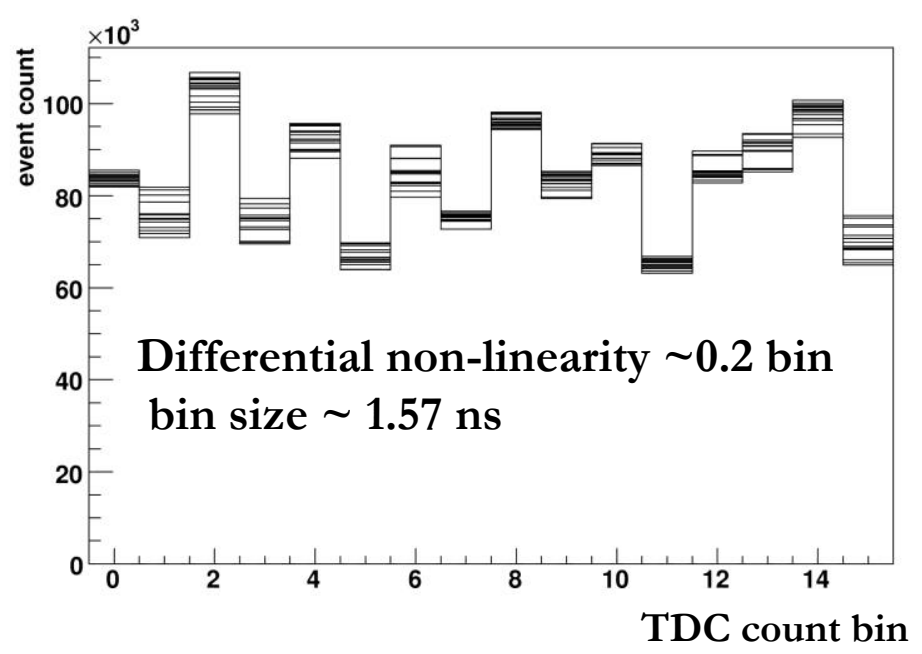


Option1 : reuse Si strip ladders.

$\checkmark$ Need to develop a new rad-hard, binary ASIC (cfr ATLAS) with $40 \mathrm{MHz}$ readout... not yet started.

Option 2 : Scintillating fibers with SiPM readout.
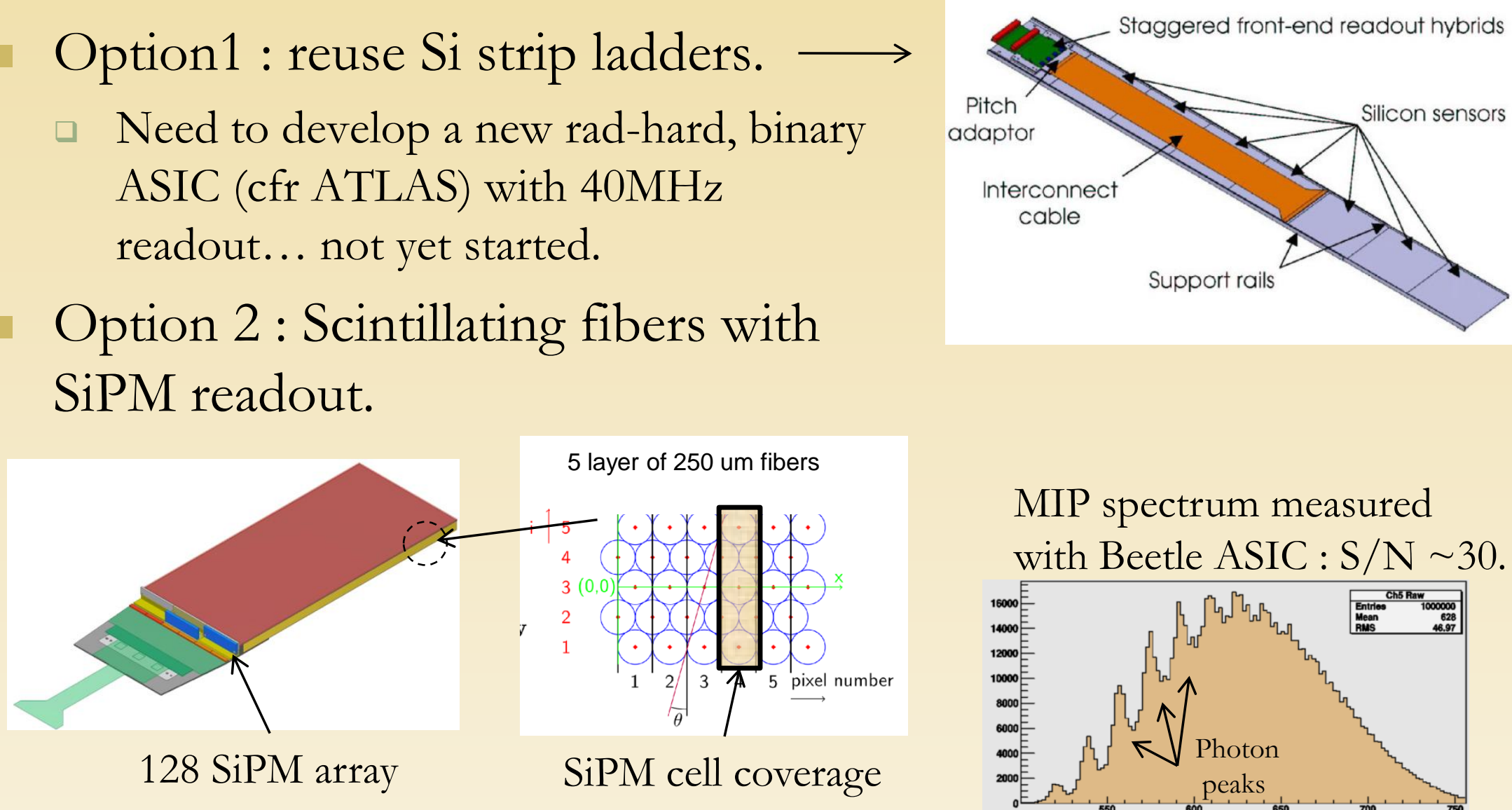

MIP spectrum measured with Beetle ASIC : S/N 30.

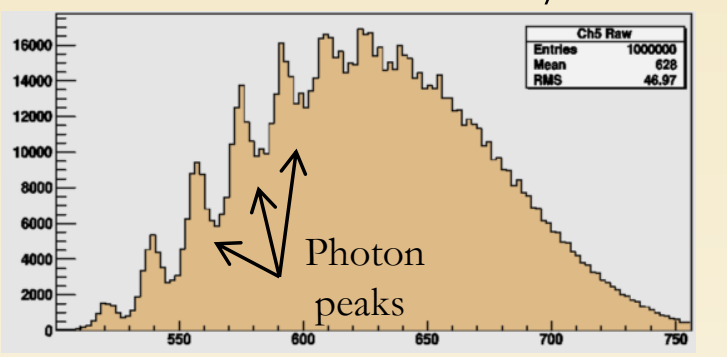

Radiation hardness for $10^{10}$ neutron $/ \mathrm{cm}^{2}$ ? under study... 


\section{Calorimeter upgrade.}

- The HV (and PMT gain) has to be decreased by a factor 5 to avoid premature ageing.

$\square \quad$->the preamplifier input equivalent noise must be decreased accordingly.

- A new frontend asic is being designed.

$\square$ The signal is alternated every $25 \mathrm{~ns}$ between two integrators. The nonative integrator is reset.

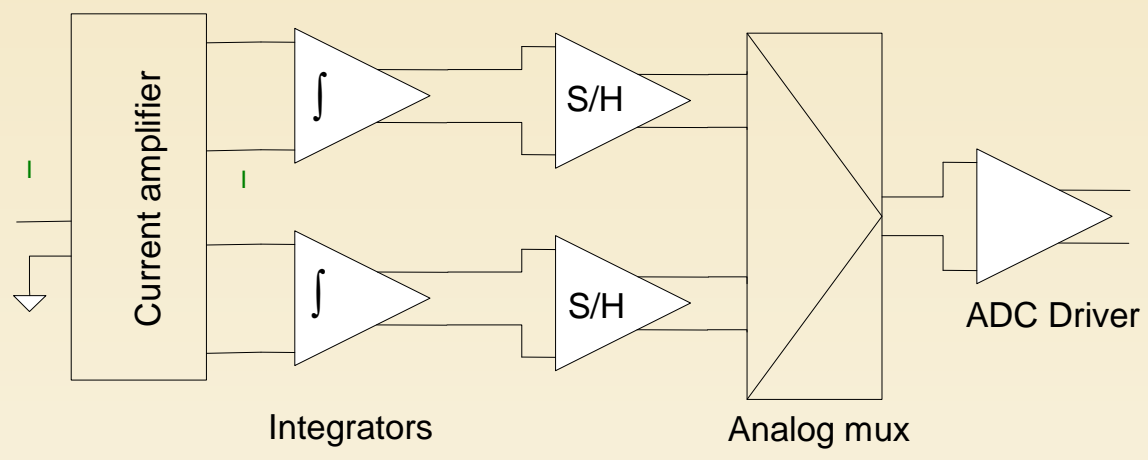

A first prototype of preamplifier and switched integrators has been designed and submitted in AMS 0.35 um SiGe BiCMOS.

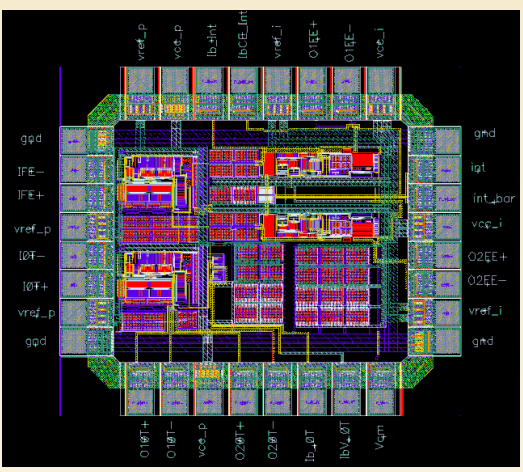




\section{Calorimeter upgrade.}

- New front end cards : 32 PMT signals

- Extensive use of FPGA's (AX and APA) for data compression, trigger and ECS

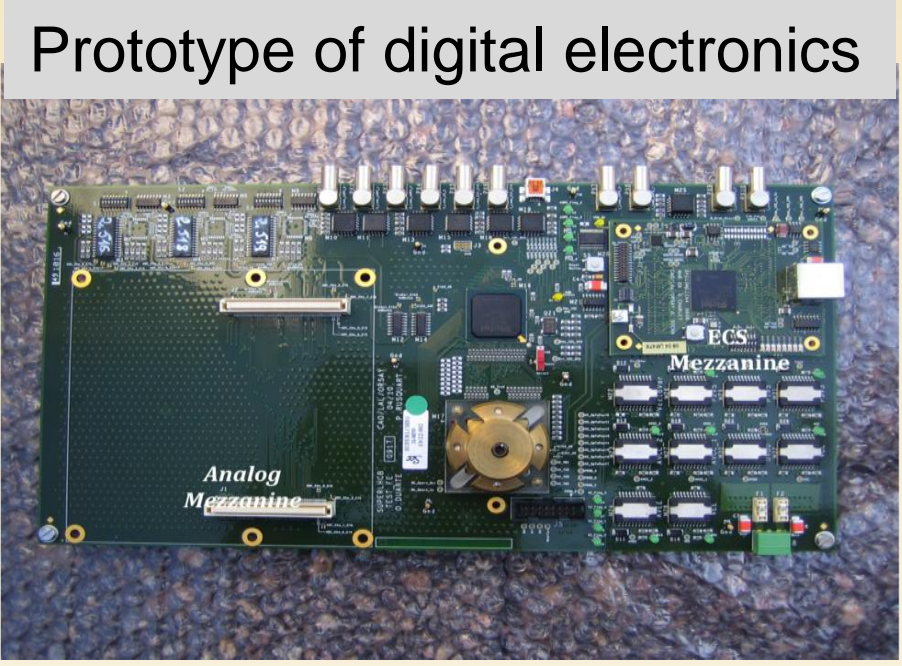

LHCb Upgrade

One Front-End block of 8 channels
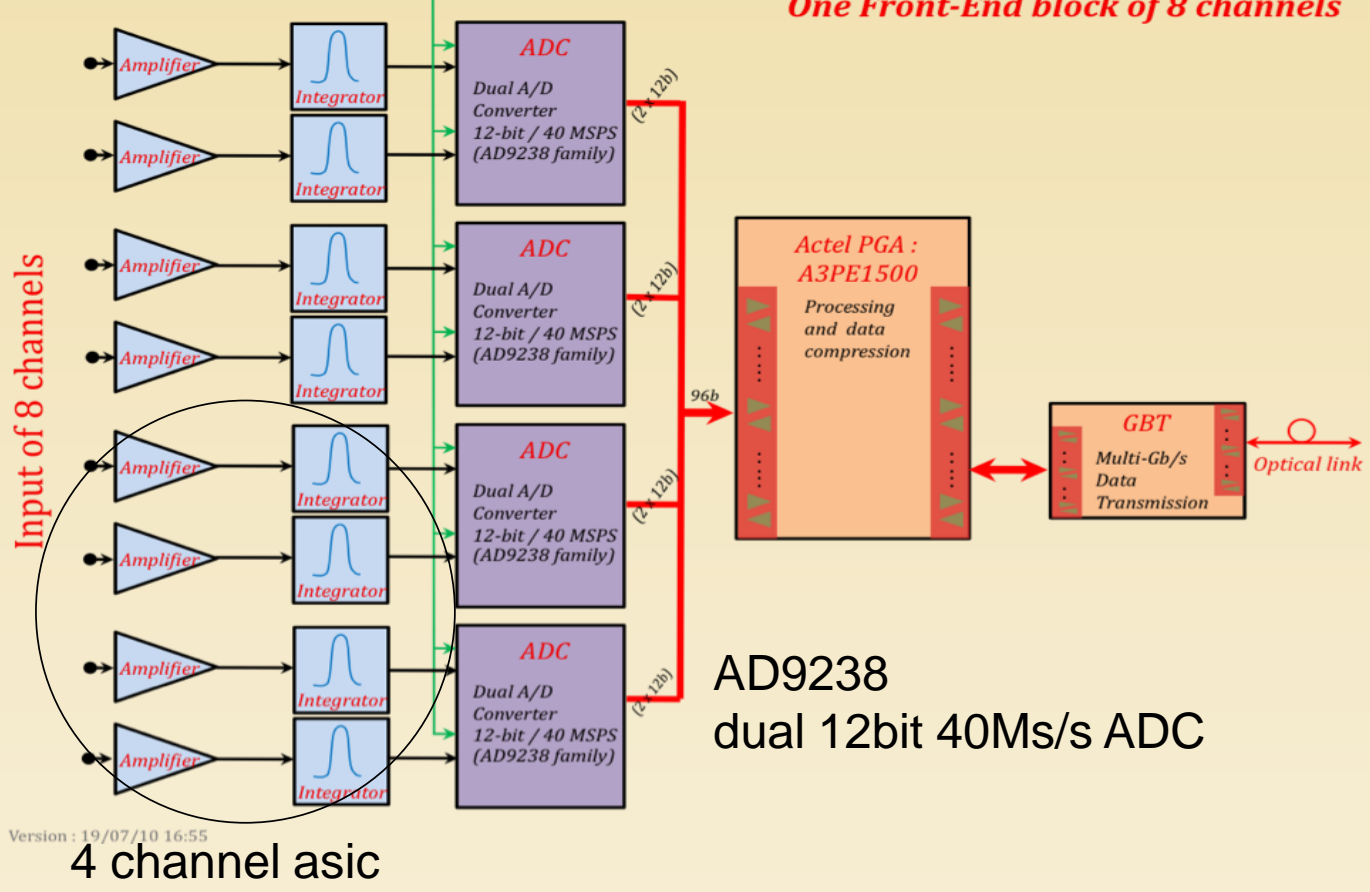

AD9238 dual 12bit 40Ms/s ADC 


\section{Muon upgrade \& Interaction trigger.}

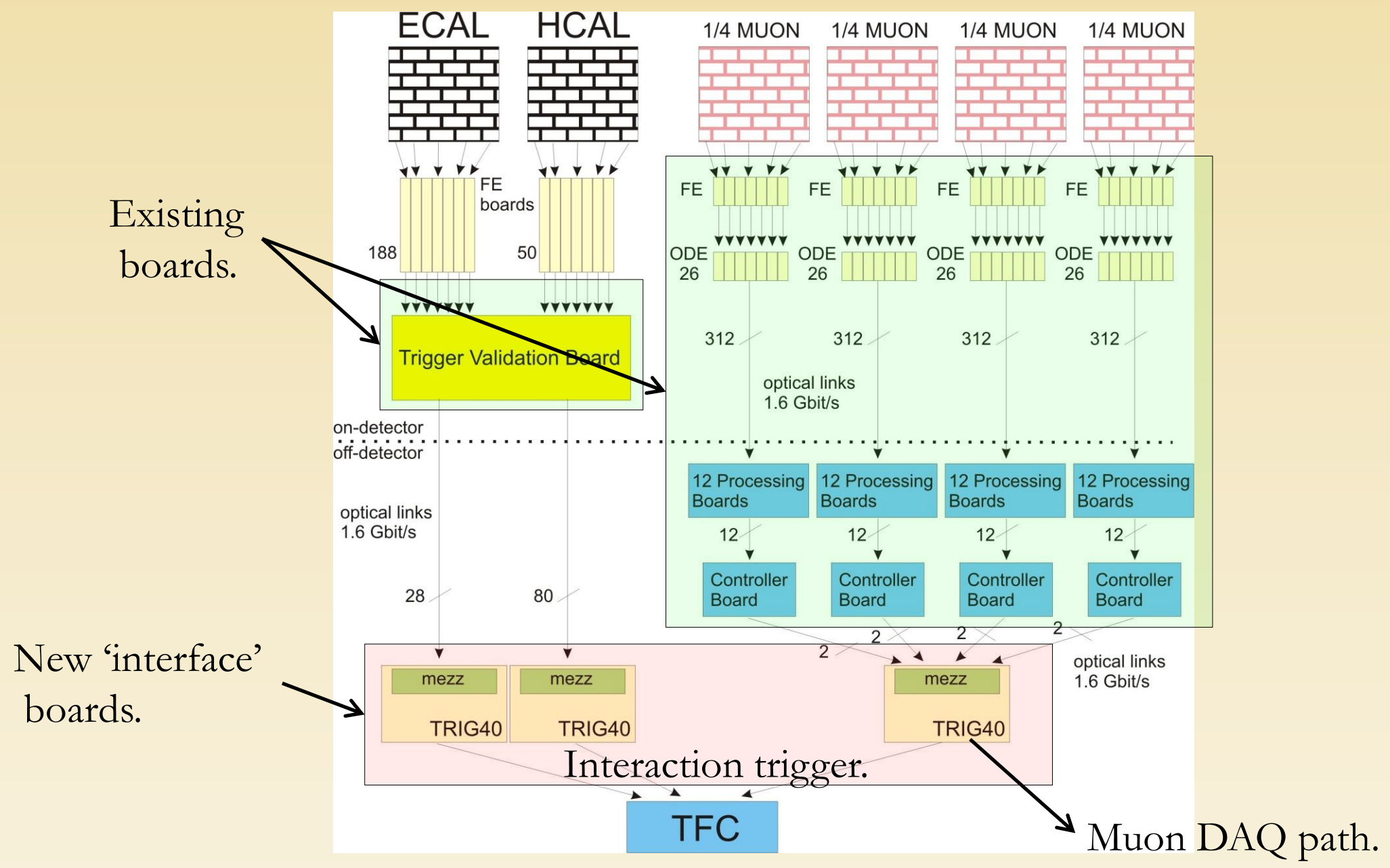


- LHCb has a firm plan for its upgrade in 2016.

- No major detector changes needed, except VELO and RICH.

- All front-end electronics must be adapted or redesigned for 40 $\mathrm{MHz}$ readout and zero suppression.

- The subdetectors electronic developments are well underway.

- Extensive use of radiation tolerant FPGA's to avoid ASIC design where possible. 


\section{Pixel matrix readout architecture.}

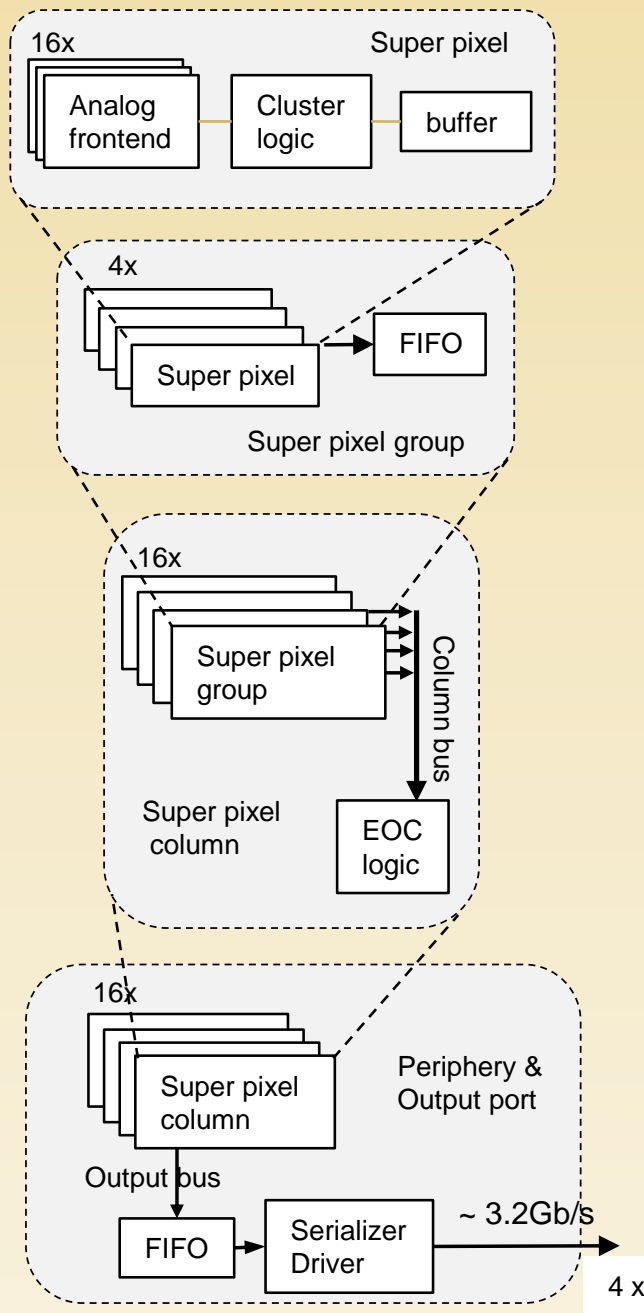

- Pixel matrix readout architecture

- Internal bus speeds:

- Column bus : 8bit@40MHz

n Output bus : 16bit@320MHz

- Total ASIC output : $\sim 12.8 \mathrm{~Gb} / \mathrm{s}$.

- Buffering in :

- Super pixel : 2 clusters

- Super pixel group FIFO : 400 bit

- Output FIFO: multi kbyte

- Simulation shows losses $<0.5 \%$ in highest occupancy conditions. 